ఠ

\title{
Identifying and assessing the risk of opioid abuse in patients with cancer: an integrative review
}

This article was published in the following Dove Press journal:

Substance Abuse and Rehabilitation

2 June 2016

Number of times this article has been viewed

\section{Ashley-Nicole Carmichael' \\ Laura Morgan' \\ Egidio Del Fabbro \\ 'School of Pharmacy, ${ }^{2}$ Division of Hematology, Oncology, and Palliative Care, Virginia Commonwealth University, Richmond, VA, USA}

Video abstract

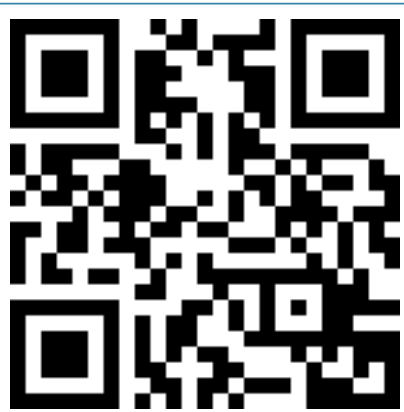

Point your SmartPhone at the code above. If you have a QR code reader the video abstract will appear. Or use: http://youtu.be/9FKYPR-_ 5U
Correspondence: Egidio Del Fabbro Division of Hematology, Oncology, and Palliative Care,Virginia Commonwealth University, I I0I East Marshall Street, Box 980230 , Richmond,VA 23298, USA Tel + I 8048289909

$\mathrm{Fax}+$ I 8248288079

Email Egidio.DelFabbro@vcuhealth.org
Background: The misuse and abuse of opioid medications in many developed nations is a health crisis, leading to increased health-system utilization, emergency department visits, and overdose deaths. There are also increasing concerns about opioid abuse and diversion in patients with cancer, even at the end of life.

Aims: To evaluate the current literature on opioid misuse and abuse, and more specifically the identification and assessment of opioid-abuse risk in patients with cancer. Our secondary aim is to offer the most current evidence of best clinical practice and suggest future directions for research.

Materials and methods: Our integrative review included a literature search using the key terms "identification and assessment of opioid abuse in cancer", "advanced cancer and opioid abuse", "hospice and opioid abuse", and "palliative care and opioid abuse". PubMed, PsycInfo, and Embase were supplemented by a manual search.

Results: We found 691 articles and eliminated 657, because they were predominantly noncancer populations or specifically excluded cancer patients. A total of 34 articles met our criteria, including case studies, case series, retrospective observational studies, and narrative reviews. The studies were categorized into screening questionnaires for opioid abuse or alcohol, urine drug screens to identify opioid misuse or abuse, prescription drug-monitoring programs, and the use of universal precautions.

Conclusion: Screening questionnaires and urine drug screens indicated at least one in five patients with cancer may be at risk of opioid-use disorder. Several studies demonstrated associations between high-risk patients and clinical outcomes, such as aberrant behavior, prolonged opioid use, higher morphine-equivalent daily dose, greater health care utilization, and symptom burden.

Keywords: screening questionnaires, urine drug screens, prescription drug-monitoring programs

\section{Introduction}

The overuse and misuse of opioid medications in the US and many other developed nations is a health crisis, leading to increased health-system utilization, emergency department visits, and nonmedical recreation use. The consequences of opioid misuse or opioid-use disorder include 16,235 deaths/year in the US alone ${ }^{1}$ because of prescriptionopioid overdose, and a rise in mortality among white middle-aged Americans for the first time in many decades, a mortality reversal that is largely attributed to death from drug and alcohol poisoning. ${ }^{2}$

There are also increasing concerns about substance abuse and diversion in patients with cancer, even at the end of life. ${ }^{3}$ A survey of palliative care programs in the US 
found less than half of respondents had policies for screening patients or their family members. ${ }^{4}$ Despite concerns, there are no guidelines from professional organizations regarding the assessment and management of opioid-use disorder in cancer. The experience derived from managing chronic noncancer pain largely determines the approach to managing opioid risk in patients with cancer. However, the evidence for managing opioid-use disorder in noncancer pain is surprisingly limited. A systematic review of 13 guidelines for chronic pain in noncancer patients found only two guidelines were of high quality. ${ }^{5}$ Despite finding limited evidence and variability in methodology, the review noted agreement on use of riskassessment tools, treatment agreements, and urine drug testing. Another systematic review of strategies to guide opioid prescribing in chronic pain found no one procedure or set of predictor variables was sufficient to identify patients at risk of opioid misuse or abuse. ${ }^{6}$ The procedures reviewed included urine toxicology screening, structured interviews, observation, and self-report questionnaires. While the concerns about opioid abuse are clearly justified, regrettably cancer pain is often undertreated in the US and Europe. ${ }^{7}$ A systematic review found one in three patients did not receive an analgesic prescription to match their level of reported pain. ${ }^{8}$

Given the current state of pain management within the oncology setting and the inherent risk of opioid abuse and misuse, the aim of this article is to evaluate the current literature on opioid abuse and more specifically the identification and assessment of opioid-abuse risk in patients with cancer. This article builds from the current state and offers the most current evidence to inform best clinical practice and future directions for research.

\section{Materials and methods}

We conducted an integrative review ${ }^{9}$ in order to broaden our approach, maintain structure, and reduce bias and errors. Our comprehensive literature search included the key terms "identification and assessment of opioid abuse in cancer", "advanced cancer and opioid abuse", "hospice and opioid abuse", and "palliative care and opioid abuse". The electronic search included PubMed, PsycInfo, and Embase, supplemented by a manual search. Articles not written in English or published before 1995 were excluded.

\section{Data evaluation}

We found 691 articles using our search terms; 657 were eliminated, because they included predominantly noncancer populations or they specifically excluded cancer patients. Studies of cancer patients on methadone maintenance or buprenorphine for substance-use disorders were not included in our final sample. There were no randomized controlled trials or systematic reviews in patients with cancer. There were several systematic reviews addressing risk in chronic nonmalignant pain, but only one review included cancer patients. That review focused on the development of opioid dependency after pain relief with opioid analgesics, ${ }^{10}$ and included a single case series of 100 cancer patients, none of whom developed any opioid dependency. ${ }^{11}$ A total of 34 articles met our criteria and were included in the final sample, including case studies, case series, retrospective observational studies, and narrative reviews. Articles available only as abstracts were also included. Ten narrative reviews addressed opioid abuse in patients with cancer. To assist in the evaluation and interpretation of the studies, a 1-point rating system was applied for relevance ( $1=$ low and $2=$ high). Articles that scored low were not excluded, but contributed less to the research aims.

\section{Data analysis}

The data were categorized as follows: studies using screening questionnaires for opioid abuse or alcohol in patients with cancer; studies using urine drug screens (UDSs) to identify opioid abuse in cancer; prescription drug-monitoring programs (PDMPs) in patients with cancer; and studies addressing the use of universal precautions (UPs) in cancer pain. Because there were few studies using screening tools specifically for opioids, we broadened our inclusion criteria to studies using the Cut Down, Annoyed, Guilty, and EyeOpener (CAGE) score in patients with cancer. In the general population, there are strong associations between early onset of alcohol use and subsequent misuse of prescription drugs. ${ }^{12}$ We excluded other addictive drugs, such as cocaine ${ }^{13,14}$ and tobacco, ${ }^{15}$ that are also associated with increased opioid misuse.

\section{Results}

Opioid-misuse or -abuse risk is identified by screening questionnaires, UDSs, PDMPs, and UPs. UPs use a combination of assessment tools, in addition to other clinical evaluations. We found one study using administrative data to identify a prior history of substance-use disorder in patients with advanced prostate cancer. A diagnosis of substance-use disorder in these patients was associated with higher mortality, hospitalization, and emergency room visits. ${ }^{16}$

\section{Screening questionnaires for opioid- misuse or -abuse risk}

Numerous validated opioid risk-screening tools exist for noncancer pain. For widespread adoption in oncology or palliative 
care clinics, opioid risk-screening tools need to be brief and easily administered. We found seven studies using opioid-risk questionnaires in patients with cancer: three using the Opioid Risk Tool (ORT), two the Screener and Opioid Assessment for Patients with Pain - revised (SOAPP-R), and two the SOAPP - short form (SOAPP-SF). The CAGE, ORT, and SOAPP-SF take less than 5 minutes to complete, and can be self-administered. There were no studies comparing opioidrisk instruments. However, some studies evaluated the association between a positive screen and other outcomes, such as aberrant behavior, increased morphine-equivalent daily dose, or abnormal UDS. We found 13 studies using the CAGE questionnaire to assess the risk of alcoholism or "chemical coping" in patients with advanced cancer (Table 1).

\section{CAGE and CAGE-AID}

CAGE is a four-item survey for identifying clinically significant alcohol problems. Although the tool is not validated for opioid-abuse risk, CAGE has been used to assess risk for chemical coping in patients with advanced cancer. ${ }^{17}$ The term "chemical coping" is often used in the cancer context, and describes patients' intake of opioids on a scale that ranges from normal nonaddictive opioid use for pain to opioid addiction. An international expert panel defines chemical coping with opioids as "The use of opioids to cope with emotional distress, characterized by inappropriate and and/or excessive opioid use". ${ }^{18}$ The CAGE - Adapted to Include Drugs (CAGE-AID) questionnaire includes drugs as well as alcohol, but has not yet been used in a cancer population. ${ }^{19}$

There was a wide variation in the prevalence of positive CAGE screens in patients with cancer, ranging from $4 \%$ to $64 \%$. This variation may be due to heterogeneity of the patient cohorts with regard to cancer type (eg, head and neck cancers associated with tobacco or alcohol use will have a higher prevalence), tumor stage, or demographics. A study of 443 cancer patients receiving palliative care in Italy found only 4\% were CAGE-“positive", ${ }^{20}$ while a Toronto cancer center identified $7 \%$ as positive (score $\geq 1 / 4$ ) in an outpatient radiotherapy clinic. ${ }^{21}$ Analgesic consumption and pain scores were not significantly higher in the positive group. Similarly, a study of cancer patients from the UK, using the Alcohol Use Disorders Identification Test, revealed few high-risk patients $(6 \%)$ and no association between a positive alcohol screen and physical or psychological symptoms. The low prevalence of high-risk patients in these three studies is in contrast to a cohort of 705 esophageal cancer patients in Germany, where $64 \%$ were CAGE-positive. ${ }^{22}$

Several retrospective studies from two North American cancer centers used the CAGE questionnaire to identify high-risk patients with advanced cancer. The prevalence of positive CAGE scores $(\geq 2 / 4)$ in a Canadian cancer center was consistent in ambulatory patients and inpatients, ranging from $24 \%^{23,24}$ to $33 \%^{25}$ in the outpatient setting, and $24 \%{ }^{26}$ to $27 \%{ }^{27}$ in the palliative care unit. The association between alcohol and opioid use-disorder risk was also reported in five studies from the MD Anderson Cancer Center. The frequency of CAGE-positive patients ranged from $17 \%{ }^{28,29}$ in patients with a variety of solid tumor types to $41 \%$ in head and neck patients on prolonged opioid therapy ( $>3$ months).$^{30}$ Other studies from the same group of investigators showed a positive CAGE score was associated with a history of illicit drug use, tobacco use, greater symptom expression, higher morphine-equivalent daily dose, and unsafe use of opioids. ${ }^{31}$ Although the efficacy of a specific intervention was not evaluated in these studies, the palliative care team was able to improve symptoms and avoid dose escalation of opioids in both positive and negative patients. ${ }^{32}$

\section{Screener and Opioid Assessment for Patients with Pain}

The SOAPP tool is a self-report questionnaire validated in chronic pain. A revised 24-item (SOAPP-R) ${ }^{33}$ version is available, and more recently a short form (SOAPP-SF) has been developed, using five questions and a Likert-type scale (0-4). We found four studies from the US using the SOAPP: two used the SOAPP-R, and two the SOAPP-SF.

A study of 38 young adults with cancer compared patients' risk based on a psychologist's evaluation and the SOAPP-R tool. ${ }^{34}$ Patients were risk-stratified, and subsequent aberrant behaviors were documented over a 9-month period. Aberrant behaviors included missed, canceled, or unscheduled appointments with a pain provider, unauthorized change in dose or frequency of a medication, hoarding drugs, and illicit drug use. Approximately $60 \%$ of patients were standard risk and demonstrated no aberrant behaviors, while $39.5 \%$ of patients were high risk and had additional safeguards placed to mitigate opioid misuse, such as frequent visits and interventions like cognitive behavioral therapy. Despite safeguards, five patients subsequently displayed aberrant behavior. Another preliminary, prospective observational study of 70 patients seen in a cancer center emergency department, found $42 \%$ were SOAP-R-positive and at high risk for aberrant behavior. ${ }^{35}$ Information obtained from the state Prescription Drug Monitoring Program (PDMP) indicated SOAPP-R-positive patients used more providers, prescriptions, and pharmacies.

Two studies of larger cohorts found a high prevalence of "positive" SOAPP-SF patients in outpatient clinics. A score $\geq 4$, considered positive and high risk for opioid 


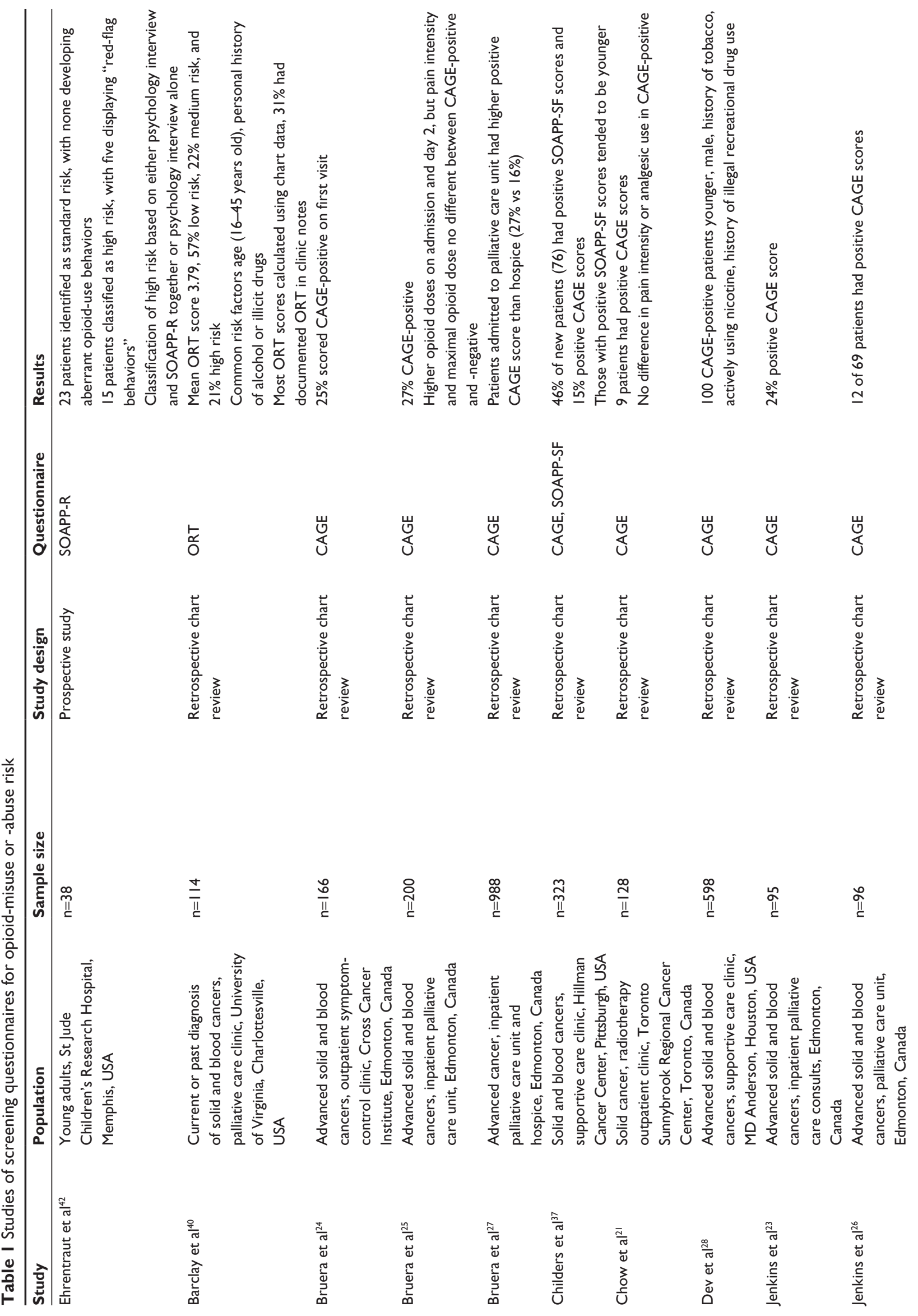



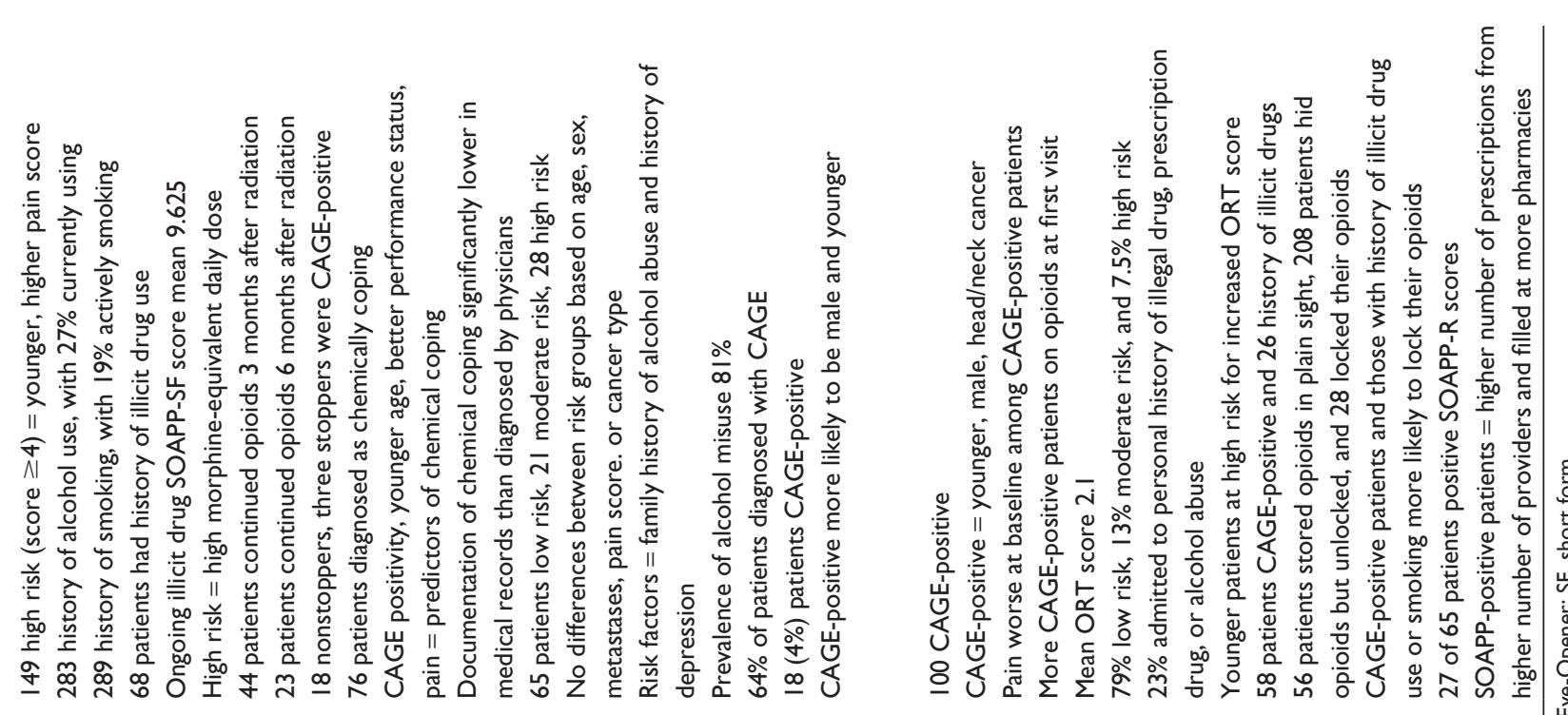

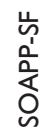

岕岕

เั

岕岕

岕

동

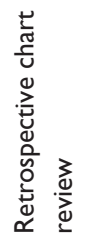

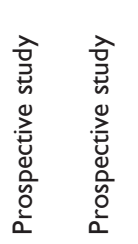

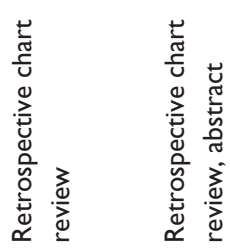

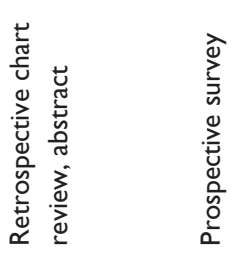

岁

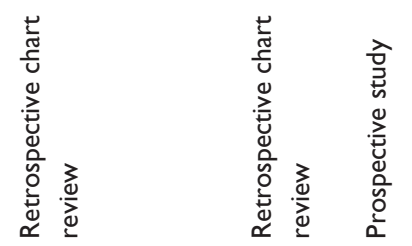

$\propto$ 닌

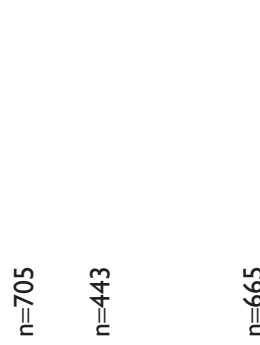

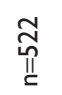

$\stackrel{ }{\stackrel{N}{*}}$

İ $\frac{0}{\frac{0}{n}}$

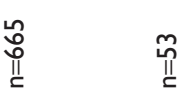

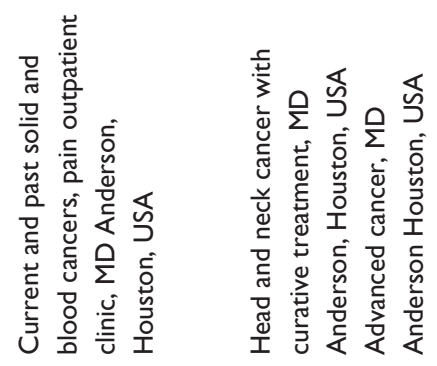

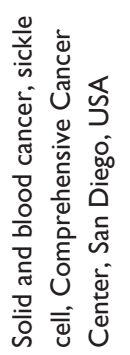

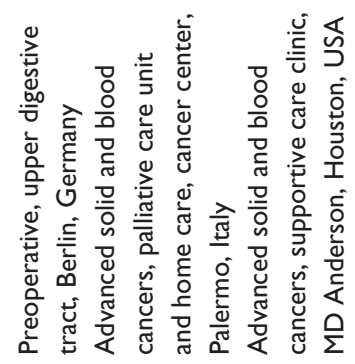

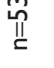

올 
misuse, was found in $29 \%$ of 522 pain clinic patients at MD Anderson Cancer Center. ${ }^{36}$ Positive patients were younger, endorsed more pain, required higher morphine-equivalent daily doses, and reported more depression and anxiety. A study in an outpatient palliative care clinic found $46 \%$ of the 57 new patients had positive SOAP-SF scores. ${ }^{37}$ Less than $5 \%$ of visits included a UDS, and $56 \%$ of these had aberrant results. Although the patients were seen in a palliative care clinic and $91 \%$ had a diagnosis of cancer, almost a third had no evidence of current disease.

\section{Opioid Risk Tool}

The ORT categorizes patients into three risk groups based on age, personal and family history of substance abuse, psychological comorbidities, and history of preadolescent sexual abuse. ${ }^{38}$ The tool can be administered and scored in less than 1 minute, and classifies patients as low, medium, or high risk for opioid misuse. We found three retrospective chart reviews using the ORT and one based on the ORT.

Three studies implemented the ORT in outpatient clinics at academic centers. A retrospective analysis of 107 patients, most with metastatic cancer, found $25 \%$ to be high risk. ${ }^{39}$ Common risk factors included a personal history of depression and family history of alcohol abuse. There was no documentation of subsequent aberrant behaviors, so it is unclear whether the tool had predictive value in this population of patients. A retrospective chart review of 114 patients with cancer assessed patients' ORT score ${ }^{40}$ and obtained UDSs in $40 \%$ of patients. Most of the ORT scores were calculated using chart data, while $31 \%$ had documentation of ORT in the clinic notes. The ORT score strongly predicted an abnormal UDS, with $62.5 \%$ of abnormal UDSs corresponding to medium- or high-risk patients. The strongest ORT predictors of abnormal UDS were family history of alcohol abuse and personal history of illegal drug use. A preliminary report from the same institution of 53 women with gynecological cancer also found younger age to be associated with a higher ORT score. ${ }^{41}$ Twenty-three percent of the women acknowledged a history of prescription or alcohol/illicit drug abuse not previously detected by routine interviews.

Pediatric clinical investigators used a checklist based on the ORT to evaluate aberrant behavior in a retrospective review of 94 adolescents and young adults (AYAs) with cancer. The Screen for Opioid-Associated Aberrant Behavior Risk (SOABR) identified a high incidence (11.7\%) of aberrant behavior in AYAs, even when opioid-prescription practices were closely monitored and multidisciplinary support was readily available. Concurrent use of multiple opioids was significantly associated with aberrant behavior $(P=0.003),{ }^{42}$ and at least one psychosocial risk factor was identified in $90 \%$. The SOABR is not yet validated, but like the ORT includes questions about patients' peer group, excessive impulsivity, and pathologic gambling. The authors note that as pediatric cancer survival rates continue to rise, survivors may face chronic cancer- or treatment-related pain that requires opioid therapy, and AYAs with aberrant behavior may be more susceptible to addiction and drug abuse later in life.

\section{Urine drug screens}

UDSs may identify drug misuse and diversion when combined with other screening measures. ${ }^{43}$ Unfortunately, there are no guidelines for using UDSs in patients with cancer, and we found only three clinical studies. Two of the studies are discussed earlier in the sections on SOAPP-SF ${ }^{37}$ and ORT. ${ }^{40}$ A third study, available as an abstract, reviewed 232 consecutive palliative pain clinic patients at a National Cancer Institute center over an 18-month period, including 80 patients who had at least one UDS. The physician's clinical judgment of substance use-disorder risk determined UDS orders. Seventy-three percent of patients tested had an abnormal UDS, either an inappropriately negative screen or positive for unprescribed substances. ${ }^{44}$ Only one in five patients had cannabis as the sole substance of misuse; most tested positive for unprescribed opioids or illicit drugs.

There are several limitations of UDSs in practice, including the interpretation of the test itself, variability of the test (different tests screen for different drugs), and variable sensitivities and specificities. In addition, one study in patients with chronic noncancer pain found an initial positive UDS did not necessarily predict aberrant behaviors once treatment started..$^{45}$ As UDS utilization increases, clinicians need to become familiar with the interpretation of abnormal results and their management. ${ }^{46}$ For the vulnerable patient with cancer who is often burdened by multiple stressors, it is especially important to avoid false accusations of opioid misuse and diversion.

\section{Prescription drug-monitoring programs}

PDMPs provide additional information that may help clinicians to identify patients at increased risk of opioid abuse. We found two pilot studies evaluating PDMPs in patients with cancer-related pain. A study from an outpatient palliative care clinic suggested that combining clinical assessment with information from a state PDMP enabled a more accurate assessment of opioid risk. ${ }^{47}$ A preliminary study published in abstract form and discussed in the SOAPP section used the PDMP to determine whether increased prescription, 
provider, and pharmacy use was associated with a positive SOAPP-R screen.

In the US, PDMPs are central statewide electronic databases that store information on prescribing and dispensing records related to federally controlled substances. ${ }^{48}$ Fifteen states require enrollment of prescribers and dispensers, while seven require only prescribers to enroll in the PDMP. ${ }^{49}$ A proposed national PDMP for Australia has highlighted the potential challenges associated with implementing "realtime" PDMPs and the need for prescribers to have access, rather than dispensers alone. ${ }^{50}$ These challenges include inadvertently directing patients toward illicit opioids, "firing" patients for misusing opioids (this would be particularly concerning in patients with a life-limiting illness, such as cancer), and inadequate pharmacological pain management. ${ }^{51}$

Reductions in prescriptions or overall opioid use after implementation of PDMPs have been inconsistent. Implementation of state PDMPs was associated with only a 3\% decrease in morphine milligram equivalents dispensed per capita $(P=0.68)$. The impact of PDMPs on morphine milligram equivalents dispensed per capita varied markedly by state: from a $66 \%$ decrease in Colorado to a $61 \%$ increase in Connecticut. ${ }^{52}$ A New York State program that mandates prescribers consult the PDMP registry before prescribing a controlled substance found a significant reduction $(P<0.05)$ in the number of opioid prescriptions and pills after implementation of the mandatory PDMP. ${ }^{53}$ Several other states recommend or encourage prescribers or dispensers to access their PDMP database. A recent survey found pharmacists frequently use the PDMP to screen for opioid misuse and doctor-shopping, but overall safe use of opioids did not improve, and PDMP users were less likely to discuss their concerns directly with the patient. ${ }^{54}$

\section{Universal precautions}

UPs in pain medicine were developed in 2005, and are a tenstep approach to the assessment and management of chronic noncancer pain, including but not limited to opioid therapy. ${ }^{55}$ Adaptations of UPs have been advocated by several narrative reviews for the treatment of cancer-related pain. ${ }^{56-57}$ Other than narrative reviews, there is no evidence in the literature that UPs improve outcomes in patients with cancer.

\section{Discussion}

In the past, oncologists and palliative care clinicians may have been reluctant to implement opioid-abuse screening, because they underappreciated the risks of addiction, diversion, and opioid-induced neurotoxicity. However, if clinicians are to alleviate the suffering of patients with cancer, manage pain, and improve quality of life, a comprehensive risk assessment for opioid abuse is essential. Opioid-use disorder in cancer is especially difficult to identify, in part because the problem may be framed in terms of "overtreatment" rather than abuse.$^{58}$ Paradoxically, despite concerns about abuse and overtreatment, many cancer patients are still not receiving opioids for severe pain, particularly in developing countries, but also in the US among minorities. ${ }^{59}$

In the absence of any standardized screening, the opioidabuse risk is likely to be underappreciated. In 1990, only $3 \%$ of inpatient and outpatient consultations by the psychiatry service at Memorial Sloan Kettering Cancer Center were related to drug abuse. ${ }^{60}$ Our review suggests the prevalence of opioid use-disorder risk is substantially higher among patients with cancer. Screening questionnaires and UDSs from five different National Cancer Institute Centers indicate at least one in five patients may be at risk of opioid-use disorder. Patients with specific tumor types related to tobacco and alcohol abuse, such as lung, esophageal, and head and neck cancers are at even greater risk, although the overall prevalence is still unclear. ${ }^{61}$ Several studies using screening questionnaires demonstrated associations between high-risk patients and important clinical outcomes, such as aberrant behavior, prolonged opioid use, higher morphine-equivalent daily dose, greater health care utilization, and symptom burden. No studies compared screening tools, although the CAGE, ORT, and SOAP-SF are all brief, easily administered questionnaires that can be adopted into daily clinical practice.

Despite concerns about implied mistrust in ordering a UDS, studies in cancer patients suggest the test should be considered depending on the clinical circumstances. However, the clinical utility, cost-effectiveness, and clinician expertise regarding interpretation are concerns that need further research. PDMPs appear to be a low-risk, low-burden, and relatively inexpensive assessment to identify aberrant behavior, such as doctor-shopping. However, the literature evidence for PDMP use in cancer patients is negligible, and the overall effect on prescription, provider, and pharmacy use is inconsistent.

Several narrative reviews advocated for the implementation of UPs. UPs were originally developed for managing nonmalignant pain, and there is no evidence UPs improve clinical outcomes in cancer patients. Despite the lack of literature evidence, UPs are appealing because of their comprehensive nature, combining assessments such as screening questionnaires, PDMPs, and UDSs along with "good" clinical practice. 
In summary, while there is a lack of standardization among providers who care for patients with cancer, current evidence supports the need for assessing opioid risk in this population. The combination of good clinical practice with guidelines for risk assessment within clinical settings is warranted. Additionally, future research should evaluate the efficacy of these opioid risk-mitigation strategies, including effects on pain control, function, and mortality. Finally, reimbursement policies, incentives, and health-technology systems that encourage physicians to use UPs, consult PDMP data, and implement screening have the potential to reduce health costs and address a serious public health problem simultaneously. ${ }^{62}$

\section{Acknowledgments}

The authors would like to thank Tara A Albrecht and John W Cyrus for their assistance in the preparation of this manuscript.

\section{Disclosure}

The authors report no conflicts of interest in this work.

\section{References}

1. Centers for Disease Control and Prevention. National Vital Statistics System: mortality data. 2015. Available from: http://www.cdc.gov/nchs/ deaths.htm. Accessed March 18, 2016.

2. Case A, Deaton A. Rising morbidity and mortality in midlife among white non-Hispanic Americans in the 21st century. Proc Natl Acad Sci US A. 2015;112(49):15078-15083.

3. Blackhall LJ, Alfson ED, Barclay JS. Screening for substance abuse and diversion in Virginia hospices. J Palliat Med. 2013;16(3): 237-242.

4. Tan PD, Barclay JS, Blackhall LJ. Do palliative care clinics screen for substance abuse and diversion? Results of a national survey. J Palliat Med. 2015;18(9):752-757.

5. Nuckols TK, Anderson L, Popescu I, et al. Opioid prescribing: a systematic review and critical appraisal of guidelines for chronic pain. Ann Intern Med. 2014;160(1):38-47.

6. Turk DC, Swanson KS, Gatchel RJ. Predicting opioid misuse by chronic pain patients: a systematic review and literature synthesis. Clin J Pain. 2008;24(6):497-508.

7. Breivik H, Cherny N, Collett B, et al. Cancer-related pain: a panEuropean survey of prevalence, treatment, and patient attitudes. Ann Oncol. 2009;20(8):1420-1433.

8. Greco M, Roberto A, Corli O, et al. Quality of cancer pain management: an update of a systematic review of undertreatment of patients with cancer. J Clin Oncol. 2014;32(36):4149-4154.

9. Whittemore R, Knafl K. The integrative review: updated methodology. J Adv Nurs. 2005;52(5):546-553.

10. Minozzi S, Amato L, Davoli M. Development of dependence following treatment with opioid analgesics for pain relief: a systematic review. Addiction. 2013;108(4):688-698.

11. Passik SD, Kirsh KL, Donaghy KB, Portenoy RK. Pain and aberrant drug-related behaviors in medically ill patients with and without histories of substance abuse. Clin J Pain. 2006;22(2):173-181.

12. McCabe SE, West BT, Morales M, Cranford JA, Boyd CJ. Does early onset of non-medical use of prescription drugs predict subsequent prescription drug abuse and dependence? Results from a national study. Addiction. 2007;102(12):1920-1930.
13. Chang G, Meadows ME, Jones JA, Antin JH, Orav EJ. Substance use and survival after treatment for chronic myelogenous leukemia (CML) or myelodysplastic syndrome (MDS). Am J Drug Alcohol Abuse. 2010;36(1):1-6.

14. Ives TJ, Chelminski PR, Hammett-Stabler CA, et al. Predictors of opioid misuse in patients with chronic pain: a prospective cohort study. BMC Health Serv Res. 2006;6:46.

15. Michna E, Ross EL, Hynes WL, et al. Predicting aberrant drug behavior in patients treated for chronic pain: importance of abuse history. J Pain Symptom Manage. 2004;28(3):250-258.

16. Chhatre S, Metzger DS, Malkowicz SB, Woody G, Jayadevappa R. Substance use disorder and its effects on outcomes in men with advanced-stage prostate cancer. Cancer. 2014;120(21):3338-3345.

17. Kwon JH, Tanco K, Park JC, et al. Frequency, predictors, and medical record documentation of chemical coping among advanced cancer patients. J Clin Oncol Oncologist. 2015;20(6):692-697.

18. Kwon JH, Hui D, Bruera E. A pilot study to define chemical coping in cancer patients using the Delphi method. J Palliat Med. 2015; 18(8):703-706.

19. Brown RL, Rounds LA. Conjoint screening questionnaires for alcohol and other drug abuse: criterion validation in a primary care practice. Wis Med J. 1995;94(3):135-140.

20. Mercadante S, Porzio G, Caruselli A, et al. The frequency of alcoholism in patients with advanced cancer admitted to an acute palliative care unit and a home care program. J Pain Symptom Manage. 2015; 49(2):254-257.

21. Chow E, Connolly R, Wong R, et al. Use of the CAGE questionnaire for screening problem drinking in an out-patient palliative radiotherapy clinic. J Pain Symptom Manage. 2001;21(6):491-497.

22. Martin MJ, Heymann C, Neumann T, et al. Preoperative evaluation of chronic alcoholics assessed for surgery of the upper digestive tract. Alcohol Clin Exp Res. 2002;26(6):836-840.

23. Jenkins CA, Schulz M, Hanson J, Bruera E. Demographic, symptom, and medication profiles of cancer patients seen by a palliative care consult team in a tertiary referral hospital. J Pain Symptom Manage. 2000;19(3):174-184.

24. Bruera E, Michaud M, Vigano A, Neumann CM, Watanabe S, Hanson J. Multidisciplinary symptom control clinic in a cancer center: a retrospective study. Support Care Cancer. 2001;9(3):162-168.

25. Bruera E, Moyano J, Seifert L, Fainsinger RL, Hanson J, SuarezAlmazor M. The frequency of alcoholism among patients with pain due to terminal cancer. J Pain Symptom Manage. 1995;10(8): 599-603.

26. Jenkins CA, Taube AW, Turner K, Hanson J, Bruera E. Initial demographic, symptom, and medication profiles in patients admitted to continuing palliative care units. J Pain Symptom Manage. 1998;16(3): 163-170.

27. Bruera E, Neumann C, Brenneis C, Quan H. Frequency of symptom distress and poor prognostic indicators in palliative cancer patients admitted to a tertiary palliative care unit, hospices, and acute care hospitals. J Palliat Care. 2000;16(3):16-21.

28. Dev R, Parsons HA, Palla S, Palmer JL, Del Fabbro E, Bruera E. Undocumented alcoholism and its correlation with tobacco and illegal drug use in advanced cancer patients. Cancer. 2011;117(19): $4551-4556$.

29. Kwon JH, Tanco K, Park JC, et al. Frequency, predictors, and medical record documentation of chemical coping among advanced cancer patients. Oncologist. 2015;20(6):692-697.

30. Kwon JH, Hui D, Chisholm G, Bruera E. Predictors of long-term opioid treatment among patients who receive chemoradiation for head and neck cancer. Oncologist. 2013;18(6):768-774.

31. Reddy A, de la Cruz M, Rodriguez EM, et al. Patterns of storage, use, and disposal of opioids among cancer outpatients. Oncologist. 2014;19(7):780-785.

32. Parsons HA, Delgado-Guay MO, El Osta B, et al. Alcoholism screening in patients with advanced cancer: impact on symptom burden and opioid use. J Palliat Med. 2008;11(7):964-968. 
33. Butler SF, Fernandez K, Benoit C, Budman SH, Jamison RN. Validation of the revised Screener and Opioid Assessment for Patients with Pain (SOAPP-R). J Pain. 2008;9(4):360-3672.

34. Anghelesc D, Ehrentraut J, Faughnan L. Opioid misuse and abuse: risk assessment and management in patients with cancer pain. J Natl Compr Canc Netw. 2013;11(8):1023-1031.

35. Wattana M, Reyes-Gibby M, Todd K. Opioid misuse risk among emergency department patients with cancer [abstract]. Ann Emerg Med. 2013 Oct;62(4 Suppl):S125.

36. Koyyalagunta D, Bruera E, Aigner C, Nusrat H, Driver L, Novy D. Risk stratification of opioid misuse among patients with cancer using the SOAPP-SF. Pain Med. 2013;14(5):667-675.

37. Childers JW, King LA, Arnold RM. Chronic pain and risk factors for opioid misuse in a palliative care clinic. Am J Hosp Palliat Care. 2015;32(6):654-659.

38. Webster LR, Webster RM. Predicting aberrant behaviors in opioidtreated patients: preliminary validation of the Opioid Risk Tool. Pain Med. 2005;6(6):432-442.

39. Ma J, Horton J, Hwang M, Atayee RS, Roeland EJ. A single-center, retrospective analysis evaluating the utilization of the Opioid Risk Tool in opioid-treated cancer patients. J Pain Palliat Care Pharmacother. 2014;28(1):4-9.

40. Barclay JS, Owens JE, Blackhall LJ. Screening for substance abuse risk in cancer patients using the Opioid Risk Tool and urine drug screen. Support Care Cancer. 2014;22(7):1883-1888.

41. Pelkofski E, Baker WD, Te Paske JR, Blackhall L, Cantrell LA. Opioid risk screening in gynecologic oncology patients: a pilot study [abstract]. Gynecol Oncol. 2015;137(Suppl 1):81.

42. Ehrentraut JH, Kern KD, Long SA, An AQ, Faughnan LG, Anghelescu DL. Opioid misuse behaviors in adolescents and young adults in a hematology/ oncology setting. J Pediatr Psychol. 2014;39(10):1149-1160.

43. Turner JA, Saunders K, Shortreed SM, et al. Chronic opioid therapy urine drug testing in primary care: prevalence and predictors of aberrant results. J Gen Intern Med. 2014;29(12):1663-1671.

44. Rauenzahn S, Del Fabbro E, Cassel J. A retrospective review of urine drug screen (UDS) findings in an outpatient palliative care clinic (S781). J Pain Symptom Manage. 2015;49(2):449-450.

45. Katz NP, Sherburne S, Beach M, et al. Behavioral monitoring and urine toxicology testing in patients receiving long-term opioid therapy. Anesth Analg. 2003;97(4):1097-1102.

46. Turner JA, Saunders K, Shortreed SM, et al. Chronic opioid therapy risk reduction initiative: impact on urine drug testing rates and results. J Gen Intern Med. 2014;29(2):305-311.

47. Muir JC, Scheffey C, Young HM, Vilches AO, Davis MS, Connor SR. Opioid prescribing practices before and after initiation of palliative care in outpatients. J Pain Symptom Manage. 2013;45(6): 1107-1111.
48. Centers for Medicare and Medicaid Services. The role of a prescription drug monitoring program reducing prescription drug diversion, misuse, and abuse. 2014. Available from: https://www.cms.gov/MedicareMedicaid-Coordination/Fraud-Prevention/Medicaid-Integrity-Education/ Downloads/drugdiversion-drugmonitoring-factsheet.pdf. Accessed March 18, 2016.

49. Prescription Drug Monitoring Program Training and Technical Assistance Center [website on the Internet]. Available from: http:// www.pdmpassist.org. Accessed March 18, 2016.

50. Islam MM, McRae IS. Real-time prescription drug monitoring program in Australia: we should be ready. Drug Alcohol Rev. 2015; 34(3):344-345.

51. Nielsen S, Bruno R. Implementing real-time prescription drug monitoring: are we ready? Drug Alcohol Rev. 2014;33(5):463-465.

52. Brady JE, Wunsch H, DiMaggio C, Lang BH, Giglio J, Li G. Prescription drug monitoring and dispensing of prescription opioids. Public Health Rep. 2014;129(2):139-147.

53. Rasubala L, Pernapati L, Velasquez X, Burk J, Ren YF. Impact of a mandatory prescription drug monitoring program on prescription of opioid analgesics by dentists. PLoS One. 2015;10(8):e0135957.

54. Green TC, Mann MR, Bowman SE, et al. How does use of a prescription monitoring program change pharmacy practice? J Am Pharm Assoc (2003). 2013;53(3):273-281.

55. Gourlay DL, Heit HA, Almahrezi A. Universal precautions in pain medicine: a rational approach to the treatment of chronic pain. Pain Med. 2015;6(2):107-112.

56. Koyyalagunta D, Burton AW, Toro MP, Driver L, Novy DM. Opioid abuse in cancer pain: report of two cases and presentation of an algorithm of multidisciplinary care. Pain Physician. 2011;14(4):E361-E371.

57. Gourlay DL, Heit HA. Universal precautions revisited: managing the inherited pain patient. Pain Med. 2009;10(Suppl 2):S115-S123.

58. Paice JA, Von Roenn JH. Under- or overtreatment of pain in the patient with cancer: how to achieve proper balance. J Clin Oncol. 2014; 32(16):1721-1726.

59. Fisch MJ, Lee JW, Weiss M, et al. Prospective, observational study of pain and analgesic prescribing in medical oncology outpatients with breast, colorectal, lung, or prostate cancer. J Clin Oncol. 2012;30(16):1980-1988.

60. Passik SD, Portenoy RK, Ricketts PL. Substance abuse issues in cancer patients. Part 1: Prevalence and diagnosis. Oncology (Williston Park). 1998;12(4):517-521, 524.

61. Rauenzahn S, Del Fabbro E. Opioid management of pain: the impact of the prescription opioid abuse epidemic. Curr Opin Support Palliat Care. 2014;8(3):273-278.

62. Katz NP, Birnbaum H, Brennan MJ, et al. Prescription opioid abuse: challenges and opportunities for payers. Am J Manag Care. 2013;19(4):295-302.

Substance Abuse and Rehabilitation

\section{Publish your work in this journal}

Substance Abuse and Rehabilitation is an international, peer-reviewed, open access journal publishing original research, case reports, editorials, reviews and commentaries on all areas of addiction and substance abuse and options for treatment and rehabilitation. The manuscript management system is completely online and includes a very quick and fair 